\title{
Rastreamento e diagnóstico ecocardiográfico das arritmias e cardiopatias congênitas fetais
}

\author{
Screening and echocardiographic diagnosis of arrhythmias and \\ congenital heart diseases in the fetus
}

Sandra Regina Marques Carvalho ${ }^{1}$, Maria Célia Mendes ${ }^{2}$, Ricardo Carvalho Cavalli ${ }^{3}$, José Cassiano Machado , Geraldo Duarte ${ }^{5}$, Aderson Tadeu Berezowski ${ }^{6}$

\section{RESUMO}

\begin{abstract}
Objetivo: analisar os resultados obtidos em programa de rastreamento e diagnóstico de arritmias e cardiopatias congênitas centrado em uma unidade terciária e determinar a importância do diagnóstico precoce na evolução fetal e neonatal. Método: foram analisados os resultados da avaliação cardíaca fetal efetuada em 1159 gestantes em dois níveis diferentes. Nível I: uso da ultra-sonografia morfológica com o objetivo de rastrear a presença de alteração cardíaca, sem estabelecer um diagnóstico diferencial. Nível II: por ecocardiograma fetal com o objetivo de diagnosticar as cardiopatias fetais existentes. Os resultados da detecção de arritmias bem como da avaliação das alterações estruturais foram comparados, sendo estabelecidas a sensibilidade e a especificidade para ambos os níveis no pré-natal, ao exame pós-natal ou à necropsia. A concordância entre ambos os níveis foi calculada pelo índice kappa. Resultados: as arritmias detectadas no nível I foram confirmadas em todos os casos e não houve falso-negativos, sendo que em cinco pacientes houve necessidade de tratamento intra-útero. A detecção das alterações estruturais obtidas no nível I teve sensibilidade de $72 \%$ e especificidade de $98 \%$, com $28 \%$ de falso-positivos. No nível II estes parâmetros foram, respectivamente, de 100 e 99\%. De acordo com o coeficiente kappa de 57\%, um grau de concordância de categoria moderada foi observado entre ambos os níveis. Das cardiopatias congênitas da nossa série $51 \%$ necessitaram intervenção farmacológica ou invasiva no período neonatal. Conclusão: o estudo ultra-sonográfico obstétrico é fundamental no rastreamento das alterações cardíacas fetais. O ecocardiograma fetal apresentou alto índice de sensibilidade e especificidade no diagnóstico das arritmias e cardiopatias congênitas, possibilitando o tratamento precoce das alterações graves.
\end{abstract}

PALAVRAS-CHAVE: Cardiopatias congênitas; Diagnóstico pré-natal; Ecocardiografia/uso diagnóstico; Doenças fetais/ ultrasonografia; Cardiopatias congênitas/ultrasonografia

\section{ABSTRACT}

Purpose: to analyze the results of a screening and diagnostic program of arrhythmias and congenital heart disease in a reference hospital and the relevance of early diagnosis in the fetal and neonate evolution. Method: cardiac evaluation of 1159 fetuses was done in two different levels. Level I: by morphological ultrasound examination with the objective to detect the existence of either arrhythmias or structural cardiac malformations. Level II: by fetal echocardiography to establish the differential diagnosis. The results of level I in the arrhythmia group were compared with those of level II, and in the group with malformations the results of both levels were confronted with the neonate echocardiogram or necropsy. The kappa index was calculated to evaluate the concordance between the two levels. Results: all detected arrhythmias in level I were confirmed in level II, there were no false negative cases and five patients with severe arrhythmia required pharmacological therapy. The diagnosis of structural malformation by level I had sensitivity of $72 \%$ and specificity of $98 \%$ and there were $28 \%$ of false-positive cases. In level II, the sensitivity and specificity of the diagnosis of congenital heart disease were 100 and $99 \%$, respectively. The kappa index was $57 \%$ and indicated a moderate degree of concordance between the two levels. Fifty-one percent of the fetuses with diagnosis of cardiac malformations required pharmacological or invasive intervention immediately after birth. Conclusion: morphological ultrasound examination is a important tool in the screening of arrhythmias and congenital heart defects during

Ambulatório de Morfologia Fetal do Departamento de Ginecologia e Obstetrícia Faculdade de Medicina de Ribeirão Preto - Universidade de São Paulo - USP Ribeirão Preto (SP), Brasil.

Pós-graduanda Bro Departamento de Ginecologia e Obstetrícia da Faculdade de Medicina de Ribeirão Preto - Universidade de São Paulo - USP - Ribeirão Pós-graduanda do
Preto (SP), Brasil.

2 Professora Doutora do Departamento de Ginecologia e Obstetrícia da Faculdade de Medicina de Ribeirão Preto - Universidade de São Paulo - USP Professora Doutora do Depart

3 Professor Doutor do Departamento de Ginecologia e Obstetrícia da Faculdade de Medicina de Ribeirão Preto - Universidade de São Paulo - USP Ribeirão Preto (SP), Brasil.

4 Professor Doutor da Faculdade de Medicina Barão de Mauá de Ribeirão Preto - (SP), Brasil.

5 Professor Titular do Departamento de Ginecologia e Obstetrícia da Faculdade de Medicina de Ribeirão Preto - Universidade de São Paulo - USP Ribeirão Preto (SP), Brasil.

6 Professor Doutor do Departamento de Ginecologia e Obstetrícia da Faculdade de Medicina de Ribeirão Preto - Universidade de São Paulo - USP Ribeirão Preto (SP), Brasil.

Correspondência: Aderson Tadeu Berezowski

Av. Bandeirantes, 3900 - 14049-900 - Ribeirão Preto - SP - Telefone: (16) 3602-2488 - e-mail: atberezo@fmrp.usp.br

Recebido em: 17/4/2006

Aceito com modificações em: 31/5/2006

Rev Bras Ginecol Obstet. 2006; 28(5):304-9 
fetal life. The sensitivity and specificity of the fetal echocardiogram were very high and the early diagnosis made it possible to treat the fetus with severe cardiac disease either during pregnancy or immediately after birth.

KEYWORDS: Congenital heart disease; Echocardiography/diagnostic use; Prenatal diagnosis; Fetal diseases/ ultrasonography; Heart defects, congenital/ultrasonography

\section{Introdução}

As cardiopatias congênitas são as malformações fetais mais freqüentes e apresentam incidência estimada de 3,5 a 12:1000 recém-nascidos vivos ${ }^{1}$. Considerando-se as perdas fetais no primeiro trimestre da gestação e os natimortos, estima-se que a incidência das cardiopatias congênitas fetais seja até cinco vezes maior que os valores $\operatorname{citados}^{2}$. Aproximadamente metade destas malformações é complexa e necessita de tratamento intervencionista após o nascimento, apresentando na sua história natural, mortalidade de 20 a $40 \%$ no período neonatal ${ }^{3}$.

As cardiopatias congênitas são de etiologia multifatorial e a maioria ocorre em gestações sem fator de risco, constituindo o rastreamento ultrasonográfico a melhor forma de detecção destas malformações antes do nascimento ${ }^{4,5}$. O rastreamento das cardiopatias fetais originou-se na França durante a década de 1980, sendo proposto como suficiente a avaliação rotineira do plano quatro câmaras cardíaco, durante a realização da ultrasonografia obstétrica ${ }^{6,7}$.

Acreditando ser esta análise básica insuficiente, foi proposta a introdução de dois niveis de avaliação cardíaca fetal ${ }^{8}$ : no nivel I, a avaliação cardíaca básica seria realizada rotineiramente por ultrasonografia obstétrica a partir do treinamento dos ultra-sonografistas com a finalidade de obtenção do plano quatro câmaras, e ao nível II seria realizado por cardiologista pediátrico com treinamento especializado em ecocardiografia fetal para revisão do diagnóstico e orientação quanto ao prognóstico e terapêutica nos casos suspeitos. Com a implantação desta proposta, taxas de detecção superiores a $80 \%$ foram inicialmente observadas em gestantes de alto risco ${ }^{9,10}$. Entretanto, estudos posteriores observaram taxas de detecção inferiores a $60 \%{ }^{11,12}$ quando esta prática se generalizou.

Em análise recente dos resultados dos programas de rastreamento observou-se que a taxa de detecção mediante a abordagem apenas do plano quatro câmaras é extremamente variável (5 a 92\%) ${ }^{5}$. $\mathrm{Na}$ verdade a avaliação isolada do plano quatro câmaras não é suficiente, especialmente quando estão presentes anomalias que comprometem a via de saída dos ventrículos e as grandes artérias, como a transposição das grandes artérias, tetralogia de Fallot, truncus arteriosus, dupla via de saída ventri- cular e interrupção do arco aórtico, pois neste plano os grandes vasos não são visualizados e o coração pode ser interpretado como normal na presença destas malformações ${ }^{13}$. Para o completo rastreamento destas malformações é necessária a abordagem de pelo menos dois outros cortes básicos, os eixos longo e curto. Mais recentemente, com a inclusão destes três planos básicos no rastreamento realizado por ultra-sonografistas, observaram-se taxas de detecção com sensibilidades superiores a $80 \% \%^{3,14}$.

O objetivo deste trabalho foi avaliar os resultados do rastreamento e diagnóstico das alterações cardiacas funcionais e estruturais em ambos os níveis, verificando a sensibilidade, a especificidade e o grau de concordância com o diagnóstico pósnatal e as conseqüências do diagnóstico na conduta fetal e do recém-nascido com cardiopatia.

\section{Métodos}

Nosso estudo baseou-se na análise retrospectiva de 1159 gestantes portadoras de fatores de risco para alterações cardíacas de seus fetos, selecionadas entre as 3061 gestantes examinadas no Ambulatório de Morfologia Fetal (AMOR) do Departamento de Ginecologia e Obstetrícia da Faculdade de Medicina de Ribeirão Preto, Universidade de São Paulo, durante o período de janeiro de 1997 a dezembro de 2004.

Estas pacientes eram provenientes dos Ambulatórios de Gestação de Alto Risco deste hospital, do próprio Ambulatório de Morfologia Fetal e de outros serviços da região. Esta população foi incluída no programa de rastreamento de cardiopatias, sendo avaliadas por exame ultra-sonográfico morfológico obstétrico e ecocardiograma fetal. Devido à perda de seguimento de 25 gestantes, a amostra populacional para análise das alterações cardiacas reduziuse a 1134 pacientes. A idade materna variou de 16 a 43 anos (média 26,4 $\pm 6,2$ ) e a idade gestacional, de 18 a 38 semanas (média 27,2 $\pm 5,4$ ).

Foram considerados como fatores de risco materno para cardiopatias fetais os antecedentes familiares de cardiopatia, idade materna superior a 35 anos, doenças crônicas como diabetes na gestação, hipertensão arterial, lúpus eritematoso, entre outras, além do uso de teratógenos e a presença de fatores fetais, alterações dismórficas fetais diagnosticadas em exame ultra-sonográfico prévio (Figura 1). 


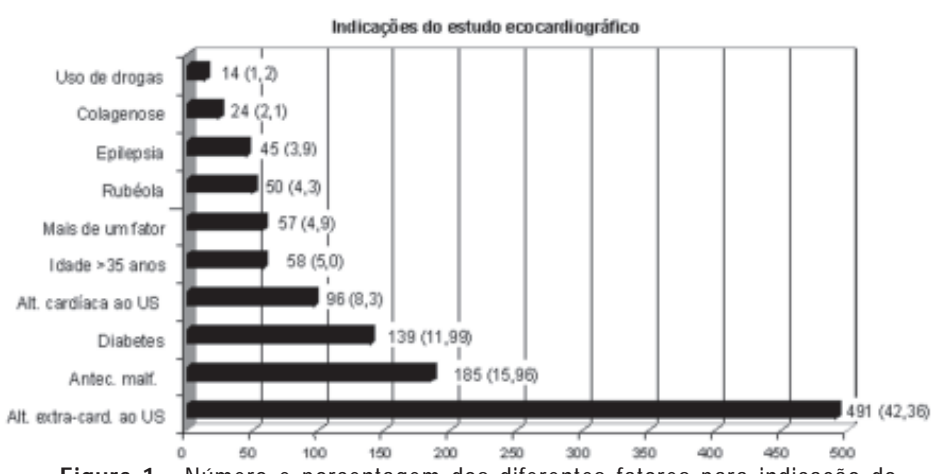

Figura 1 - Número e porcentagem dos diferentes fatores para indicação do ecocardiograma fetal.

Antec: antecedentes; Malf: malformações; Alt: alteração; Card: cardíaca; US: ultra-sonografia.

As alterações cardiacas foram divididas em dois tipos: funcionais (arritmias) e estruturais. Devido à possibilidade de as arritmias serem transitórias, considerou-se como incidência real a obtida no ecocardiograma fetal, enquanto a incidência real das alterações estruturais foi determinada pelo diagnóstico ecocardiográfico pós-natal ou à necropsia (Figuras 2 e 3).

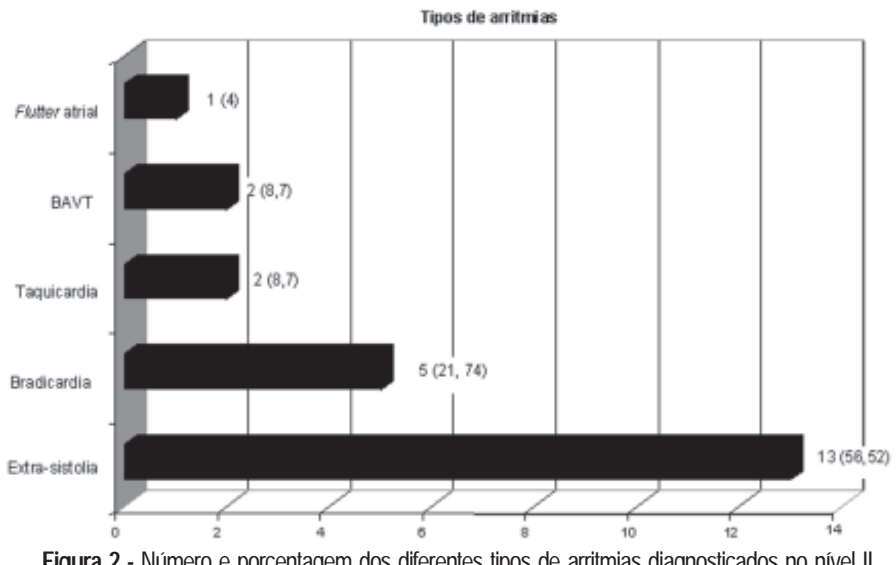

Figura 2 - Número e porcentagem dos diferentes tipos de arritmias diagnosticados no nível II. BAVT = bloqeio atrioventricular total.

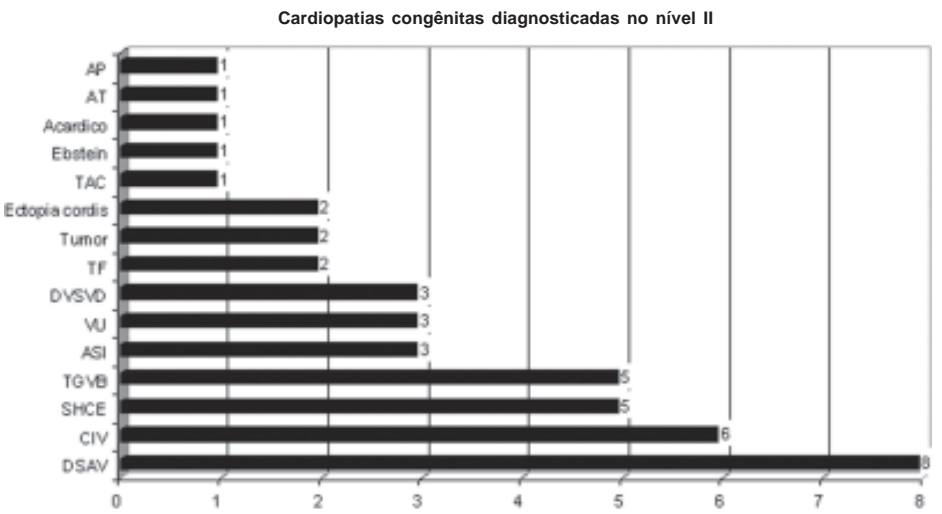

Figura 3 - Número de casos das diferentes cardiopatias congênitas diagnosticadas no nível II. DSAV: defeito do septo atrioventricular; CIV: comunicação interventricular; SHCE: síndrome de hipoplasia do coração esquerdo, TGVB: transposição dos grandes vasos da base, ASI: aneurisma do septo interatrial; VU: ventrículo único; DVSVD: dupla via de saída do ventrículo direito; TF: tetralogia de Fallot; TAC: truncus arteriosus comunis, AT: atresia tricúspide; AP: atresia pulmonar.
O rastreamento ultra-sonográfico (nivel I) foi realizado por ultra-sonografista do serviço e consistiu na avaliação de todos os órgãos e sistemas pela técnica habitual e do sistema cardiovascular mediante a obtenção dos cortes básicos (quatro câmaras, eixo longo e eixo curto) e determinação da freqüência e ritmo cardíaco mediante a análise do Modo M. Neste nível o objetivo foi estabelecer a presença de alteração do ritmo cardíaco ou de alteração estrutural, sem necessidade de definir especificamente o tipo de malformação.

O estudo ecocardiográfico (nível II) foi realizado por ecocardiografista especializado na avaliação cardíaca fetal, imediatamente a seguir do exame do nivel I, abordando exclusivamente o sistema cardiovascular, com a obtenção dos seguintes cortes: corte transverso abdominal para determinar o situs visceral pela posição da aorta e da veia cava inferior em relação à coluna vertebral, corte quatro câmaras para avaliação das quatro cavidades cardíacas e da junção atrioventricular e cortes eixo longo e eixo curto para demonstrar as relações espaciais e conexões dos átrios com os ventrículos e destes com os vasos da base do coração e a bifurcação da artéria pulmonar (Figura 4). O estudo da dopplerfluxometria do coração foi avaliado por este nível, porém esta análise não foi considerada na avaliação conjunta dos dois níveis. Neste nível, o objetivo foi estabelecer o diagnóstico específico do tipo de malformação tanto nas alterações estruturais quanto nas arritmias.

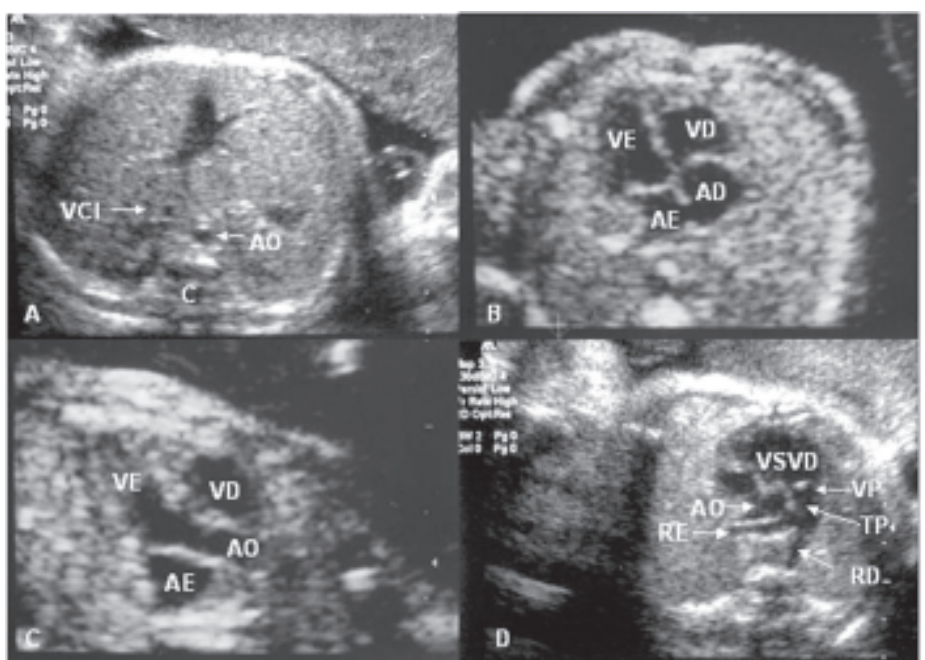

Figura 4 - Os quatro planos ecocardiográficos básicos. A - Transverso abdominal: permite determinar 0 situs visceral pela posição da aorta e veia cava inferior em relação à coluna vertebral. B - Quatro câmaras: possibilita a visibilização das quatro cavidades cardíacas e da junção atrioventricular. C - Eixo longo: demonstra a relação e a conexão do átrio esquerdo com o ventrículo esquerdo e deste com a aorta. Situado anteriormente ao ventrículo esquerdo observa-se 0 ventrículo direito e entre ambos o septo interventricular e sua continuidade com a parede anterior da aorta. D - Eixo curto: corte transverso na altura da valva aórtica que ocupa o centro do plano. Envolvendo a aorta observa-se a via de saída do ventrículo direito, a valva pulmonar, o tronco de artéria pulmonar e a sua bifurcação nos ramos direito e esquerdo. AD: átrio direito; $A E$ : átrio esquerdo; $A O$ : aorta; $C$ : coluna vertebral; RD: ramo direito da artéria pulmonar; RE: ramo esquerdo da artéria pulmonar; TP: tronco da artéria pulmonar; VCl: veia cava inferior; VD: ventrículo direito; VE: ventrículo esquerdo; VP: valva pulmonar; VSVD: via de saída do ventrículo direito. 
As arritmias foram classificadas em benignas e graves. O primeiro grupo compreende as alterações da freqüência sinusal e as extrassístoles atriais e ventriculares, e o segundo grupo, as taquiarritmias supra e ventriculares, o flutter e a fibrilação atrial e os bloqueios atrioventriculares de grau avançado. Esta classificação foi fundamentada na possibilidade de descompensação cardíaca, hidropisia fetal e óbito.

Os exames de ultra-sonografia obstétrica e ecocardiograma fetal foram realizados sucessivamente em equipamentos ATL (Advanced Technology Laboratories), modelos HDI (High Definition Image) 3000 e 3500 , com modalidades ultra-sonográficas de Modo M, bidimensional de alta definição e Doppler pulsado de potência e em cores, utilizando-se transdutores convexos multi-frequenciais de 4 e $8 \mathrm{MHz}$.

\section{Resultados}

\section{Nivel I}

Foram detectadas 23 alterações funcionais (arritmia cardíaca), correspondendo a $2 \%$ das gestantes avaliadas, e todas foram confirmadas no nível II, não havendo falso-negativos. Foram detectadas alterações estruturais em 51 fetos, sendo confirmadas em 28 (55\%) no exame pós-natal ou à necropsia, enquanto 23 (45\%) não apresentavam alteração cardiovascular (falso-positivos). Nos 28 casos que apresentavam alteração estrutural, a análise do plano quatro câmaras foi fundamental para o sucesso na detecção em 21 (75\%) e a dos outros cortes na detecção de sete (25\%). Nos 23 casos falso-positivos a detecção foi fundamentada na análise do plano quatro câmaras em 21 (91\%) e do eixo longo ou curto em dois (9\%). Houve 11 casos falsonegativos (não detectados neste nível) que corresponderam a $28 \%$ dos 39 fetos com alterações estruturais confirmadas. Considerando-se os planos ecocardiográficos básicos, a análise do plano quatro câmaras foi determinante em 4/11 (36\%) e dos demais cortes em 7/11 (64\%).

A análise estatística demonstrou que os resultados deste nível apresentaram sensibilidade de $72 \%$ com intervalo de confiança em 95\% (IC 95\%) variando entre 55 e 85\% (IC 95\% = 55 a 85\%) e especificidade em 98\% com IC 95\% variando entre 96 e 98\% (IC 95\% = 96 a 98\%) em relação ao diagnóstico pós-natal (Tabela 1).

Tabela 1 - Estimativas de sensibilidade e especificidade para o rastreamento no nível e no nível II.

\begin{tabular}{lcc}
\hline Método & Sensibilidade & Especificidade (\%) \\
\hline Nível I & $0,718(0,551-0,850)$ & $0,979(0,969-0,987)$ \\
Nível II & $1,000(0,910-1,000)$ & $0,995(0,989-0,998)$ \\
\hline
\end{tabular}

\section{Nivel II}

Neste nivel foram confirmadas as 23 alterações funcionais diagnosticadas no nível I; destas, $18(78 \%)$ foram consideradas arritmias benignas e cinco (22\%) graves. Os tipos de arritmias diagnosticadas estão expostos na Figura 2.

Foram diagnosticadas 44 alterações estruturais (Figura 3) e 39 (87\%) foram confirmadas no período neonatal por estudo ecocardiográfico ou à necropsia. Houve cinco casos falso-positivos que corresponderam a três comunicações interventriculares e dois aneurismas do septo interatrial (Figura 3). Considerando-se os planos ecocardiográficos utilizados no diagnóstico destas cardiopatias, o plano quatro câmaras foi determinante em três casos (dois aneurismas do septo interatrial e um de comunicação interventricular) e o eixo longo em dois (duas comunicações interventriculares). Não houve no estudo ecocardiográfico diagnósticos falso-negativos de cardiopatia estrutural.

Entre os 39 fetos com diagnóstico confirmado de cardiopatia estrutural, somente três $(8 \%)$ apresentavam fatores de risco materno para incidência de cardiopatias congênitas e 36 (93\%) apresentavam fator de risco fetal. A análise estatística dos resultados deste nível evidenciou sensibilidade de $100 \%$ com IC 95\% variando entre 91 e $100 \%$ (IC 95\% = 91 a 100\%) e especificidade de 99,5\% com IC 95\% variando entre 98 e 99\% (IC 95\% = 98 a 99\%) do nível II em relação ao diagnóstico pós-natal (Tabela 1).

Para determinar o grau de concordância e minimizar a influência do acaso na interpretação dos dados entre o diagnóstico de ambos os níveis, foi realizado o teste kappa obtendo-se o coeficiente $57 \%$ com IC $95 \%$ variando entre 45 e $69 \%$ (IC $95 \%=45$ a $69 \%)$. Com base na categorização proposta por Landis and $\mathrm{Koch}^{15}$ (0,00-0,20: desprezível; 0,21-0,40: mínima; 0,41- 0,60: moderada; 0,61080: grande e 0,81-1,00: perfeita), obteve-se força de concordância de 0,57 (moderada) entre os dois níveis de diagnóstico (Tabela 2).

Tabela 2 - Concordância entre o nível I e o nível II pelo índice kappa.

\begin{tabular}{lcrrrr}
\hline Nível I & \multicolumn{2}{c}{ Nível II } & Total & kappa & IC 95\% \\
& Positivo & Negativo & & & \\
\hline Positivo & 28 & 23 & 51 & 0,572 & $(0,451-0,693)$ \\
Negativo & 16 & 1067 & 1083 & & \\
Total & 44 & 1090 & 1134 & & \\
\hline
\end{tabular}

IC $95 \%$ = intervalo de confiança a $95 \%$.

Houve necessidade de intervenção terapêutica no período pré e pós-natal em cinco casos com diagnóstico de arritmia grave (22\%) e de procedimentos intervencionistas no período neonatal em 20 casos com cardiopatia estrutural (51\%). 


\section{Discussão}

O rastreamento ultra-sonográfico das cardiopatias congênitas fetais assumiu grande importância nas últimas duas décadas e representa atualmente o principal método de detecção pré-natal destas alterações. Deve ser realizado entre a $24^{\mathrm{a}}$ e a $28^{\text {a }}$ semana da gestação pela maior facilidade de obtenção dos cortes ecocardiográficos necessários ${ }^{16}$.

No segundo trimestre é também possivel o diagnóstico de determinadas cardiopatias evolutivas que podem estar ausentes no primeiro trimestre. A análise da influência da idade gestacional sobre o rastreamento das cardiopatias mostra que a taxa de detecção destas doenças, quando obtidas entre a $16^{\mathrm{a}}$ e a $28^{\mathrm{a}}$ semana, é $17 \%$ maior do que quando as análises são realizadas no primeiro trimestre, devido à existência de anomalias evolutivas $^{17}$. No presente estudo, o resultado que obtivemos com idade gestacional média de 27 semanas pode ter sido determinado pela elevada taxa de sensibilidade do rastreamento e do ecocardiograma fetal em relação ao diagnóstico pós-natal.

Em avaliação realizada em 20 centros europeus, observou-se que o rastreamento obtido pelo nível I apresenta taxas de detecção que variam entre 8 a $48 \%$, de acordo com o grau de treinamento dos obstetras e ultra-sonografistas na obtenção dos cortes ecocardiográficos básicos ${ }^{18}$.

Na nossa experiência, no nível I a taxa de sucesso na detecção das cardiopatias congênitas foi de $72 \%$. Analisando-se este resultado em relação à importância da obtenção dos planos ecocardiográficos, foi evidente que o corte quatro câmaras foi mais facilmente obtido, já que em $75 \%$ das alterações estruturais confirmadas, este plano foi fundamental para o diagnóstico. Entretanto, os ultra-sonografistas ainda encontram dificuldade para a análise correta deste plano, uma vez que em 91\% dos falso-positivos a detecção de cardiopatia estrutural foi baseada na análise deste corte. Possivelmente esta dificuldade deve-se às peculiaridades da anatomia cardíaca e à falta de parâmetros ultra-sonográficos simples de mensuração destas câmaras, a exemplo do estudo de outros órgãos, como o tamanho do ventrículo cerebral, da cisterna magna, da circunferência abdominal, fêmur, entre outros ${ }^{5}$.

Na nossa série a inclusão dos cortes eixo longo e eixo curto na avaliação do nível I foram determinantes para a detecção de $25 \%$ das alterações estruturais e pode explicar a sensibilidade de $72 \%$, em concordância com outros estudos importantes que obtiveram taxas de detecção superiores a $70 \%{ }^{13,14}$.
No nível II, o baixo índice de falso-positivos e a ausência de falso-negativos demonstraram a acurácia deste exame no diagnóstico das cardiopatias congênitas. Houve cinco casos falso-positivos, sendo dois aneurismas do septo interatrial e três comunicações interventriculares. A discordância diagnóstica, nestes casos, pode ser explicada pela possibilidade de regressão destes defeitos. O crescimento do tecido septal ou o acolamento do tecido subtricuspídeo podem ocluir uma comunicação interventricular ainda intra-útero ou no período pósnatal $^{20}$. De forma semelhante, o aumento da espessura septal e a redução da resistência pulmonar após o nascimento podem reduzir a movimentação exagerada do septum primum que caracteriza o aneurisma, fazendo com que este defeito desapareça após o nascimento ${ }^{20}$.

As arritmias fetais benignas corresponderam a $90 \%$ das alterações do ritmo diagnosticadas e são representadas por alterações da freqüência sinusal e por extra-sístoles isoladas ${ }^{21}$. Estas arritmias são geralmente transitórias e respondem a medidas terapêuticas como modificação de hábitos ou supressão de drogas. As arritmias graves correspondem a menos de $10 \%$ das arritmias fetais, porém estas podem acarretar grave comprometimento hemodinâmico ${ }^{22}$. Na nossa série as arritmias benignas corresponderam a $78 \%$ e as arritmias graves a $22 \%$.

No nível I, o objetivo do ultra-sonografista foi detectar a presença de alterações do ritmo cardíaco sem determinar o diagnóstico diferencial da arritmia. A simplicidade deste procedimento permitiu rastreamento eficaz das arritmias como comprovado pela taxa de detecção de $100 \%$ e a ausência de falso-negativos.

No nível II, cinco das 23 arritmias diagnosticadas foram consideradas graves, sendo dois casos de taquicardia supraventricular sustentada, um flutter atrial e dois casos de bloqueio atrioventricular. Os três primeiros casos receberam tratamento intra-útero com reversão da arritmia. Dos casos com bloqueio atrioventricular, um apresentou hidropisia fetal precocemente e evoluiu a óbito e o outro, hemodinamicamente compensado, foi tratado intra-útero com isoproterenol e dexametosona, evoluindo estável até o nascimento, quando foi implantado marca-passo nos primeiros dias de vida.

A ausência de fatores de risco materno em $92 \%$ dos fetos com cardiopatia confirmou que as alterações morfológicas ultra-sonográficas fetais foram o principal fator indicativo da ocorrência destas malformações e demonstra a importância do rastreamento e da indicação do ecocardiograma fetal no diagnóstico específico destas alterações. 
O rastreamento das alterações cardiovasculares no nível I possibilitou a detecção de todas as arritmias e de 55\% das cardiopatias congênitas mediante a avaliação do plano quatro câmaras, e a inclusão dos cortes eixo longo e curto contribuiu para a obtenção de taxas de sensibilidade e especificidade semelhantes a outros estudos em que este procedimento foi adotado.

As taxas elevadas de sensibilidade e especificidade do nível II comparadas ao diagnóstico pósnatal demonstraram que o ecocardiograma fetal é fundamental no diagnóstico pré-natal das cardiopatias congênitas.

Portanto, apesar das diferenças obtidas entre os niveis I e II, houve complementação entre ambos, o que justifica a utilização destes métodos em um centro terciário, o qual deve poder disponibilizar os recursos mais sofisticados para o atendimento especializado.

\section{Agradecimentos}

Aos profissionais Adriana de Fátima Lourenço e Roberto Molina Souza, integrantes do Centro de Métodos Quantitativos da Faculdade de Medicina de Ribeirão Preto da Universidade de São Paulo - USP (CEMEQ), pela realização da análise estatística dos dados apresentados neste trabalho.

\section{Referências}

1. Hoffman JI. Incidence of congenital heart disease: I. Postnatal incidence. Pediatr Cardiol. 1995;16(3):103-13.

2. Hoffman JI. Incidence of congenital heart disease: II Prenatal incidence. Pediatr Cardiol. 1995;16(4):155-65.

3. Allan L, Benacerraf B, Copel JA, Carvalho JS, Chaoui $\mathrm{R}$, Eik-Ness $\mathrm{SH}$, et al. Isolated major congenital heart disease. Ultrasound Obstet Gynecol. 2001;17(5):370-9

4. Allan LD, Sharland GK, Milburn A, Lockhart SM, Groves AM, Anderson RH, et al. Prospective diagnosis of 1,006 consecutive cases of congenital heart disease in the fetus. J Am Coll Cardiol. 1994;23(6):1452-8.

5. Chaoui R. The four-chamber view: four reasons why it seems to fail in screening for cardiac abnormalities and suggestions to improve detection rate. Ultrasound Obstet Gynecol. 2003;22(1):3-10.

6. Allan L. Antenatal diagnosis of heart disease. Heart. 2000;83(3):367-70.

7. Lopes LM, Damiano AP, Zugaib M. Programa educativo de treinamento em ecocardiografia fetal nível I: impacto na referência e análise de resultados. Rev Bras Ecocardiogr. 2003;16(3):61-8.

8. DeVore GR. The prenatal diagnosis of congenital heart disease - a practical approach for the fetal sonographer. J Clin Ultrasound.1985;13(4):229-45.

9. Copel JA, Pilu G, Green J, Hobbins JC, Kleinman CS. Fetal echocardiographic screening for congenital heart disease: the importance of the four-chamber view. Am J Obstet Gynecol. 1987;157(3):648-55.

10. Bromley B, Estroff JA, Sanders SP, Parad R, Roberts $\mathrm{D}$, Frigoletto FD Jr, et al. Fetal echocardiography: accuracy and limitations in a population at high and low risk for heart defects. Am J Obstet Gynecol. 1992;166(5):1473-81.

11. Buskens E, Grobbee DE, Frohn-Mulder IM, Stewart PA, Juttmann RE, Wladimiroff JW, et al. Efficacy of routine fetal ultrasound screening for congenital heart disease in normal pregnancy. Circulation. 1996;94(1):67-72.

12. Fernandez CO, Ramaciotti C, Martin LB, Twickler DM. The four-chamber view and its sensitivity in the detecting congenital heart defects. Cardiology. 1998;90(3):202-6.

13. Achiron R, Glaser J, Gelernter I, Hegesh J, Yagel S. Extended fetal echocardiographic examination for detecting cardiac malformations in low risk pregnancies. BMJ. 1992;304(6828):671-4.

14. Carvalho JS, Mavrides E, Shinebourne EA, Campbell $\mathrm{S}$, Thilaganathan B. Improving the effectiveness of routine prenatal screening for major congenital heart defects. Heart. 2002;88(4):387-91.

15. Landis JR, Koch GG. The measurement of observer agreement for categorical data. Biometrics. 1977;33(1):159-74.

16. Allan L. Technique of fetal echocardiography. Pediatr Cardiol. 2004;25(3):223-33.

17. Yagel S, Weissman A, Rotstein Z, Manor M, Hegesh J, Anteby E, et al. Congenital heart defects: natural course and in utero development. Circulation. 1997;96(2):550-5.

18. Garne E, Stoll C, Clementi M; Euroscan Group. Evaluation of prenatal diagnosis of congenital heart diseases by ultrasound: experience from 20 European registries. Ultrasound Obstet Gynecol. 2001;17(5):386-91.

19. Paladini D, Palmiere S, Lamberti A, Teodoro A, Martinelli P, Nappi C. Characterizacion and natural history of ventricular septal defects in the fetus. Ultrasound Obstet Gynecol. 2000;16(2):118-22.

20. Papa M, Fragasso G, Camesasca C, Di Turi RP, Spagnolo D, Valsecchi L, et al. Prevalence and prognosis of atrial septal aneurysm in the high risk fetus without structural heart defects. Ital Heart J. 2002;3(5):318-21.

21. Kleinman CS, Nehgme RA. Cardiac arrhythmias in the human fetus. Pediatr Cardiol. 2004;25(3):234-51.

22. Strasburger JF. Fetal arrhythmias. Prog Pediatr Cardiol. 2000;11(1):1-17. 